\title{
Geographical distribution of Anopheles stephensi in eastern Ethiopia
}

\author{
Meshesha Balkew ${ }^{1 *}$, Peter Mumba ${ }^{1}$, Dereje Dengela ${ }^{2}$, Gedeon Yohannes ${ }^{1}$, Dejene Getachew ${ }^{3}$, Solomon Yared ${ }^{4}$, \\ Sheleme Chibsa ${ }^{5,6}$, Matthew Murphy ${ }^{5,7}$, Kristen George ${ }^{5,8}$, Karen Lopez ${ }^{9}$, Daniel Janies ${ }^{9}$, Sae Hee Choi $^{10}$, \\ Joseph Spear ${ }^{10}$, Seth R. Irish ${ }^{5,7}$ and Tamar E. Carter ${ }^{10}$
}

\begin{abstract}
Background: The recent detection of the South Asian malaria vector Anopheles stephensi in Ethiopia and other regions in the Horn of Africa has raised concerns about its potential impact on malaria transmission. We report here the findings of a survey for this species in eastern Ethiopia using both morphological and molecular methods for species identification.

Methods: Adult and larval/pupal collections were conducted at ten sites in eastern Ethiopia and Anopheles specimens were identified using standard morphological keys and genetic analysis.

Results: In total, 2231 morphologically identified An. stephensi were collected. A molecular approach incorporating both PCR endpoint assay and sequencing of portions of the internal transcribed spacer 2 (ITS2) and cytochrome $c$ oxidase subunit 1 (cox1) loci confirmed the identity of the An. stephensi in most cases (119/124 of the morphologically identified An. stephensi confirmed molecularly). Additionally, we observed Aedes aegypti larvae and pupae at many of the An. stephensi larval habitats.
\end{abstract}

Conclusions: Our findings show that An. stephensi is widely distributed in eastern Ethiopia and highlight the need for further surveillance in the southern, western and northern parts of the country and throughout the Horn of Africa.

Keywords: Anopheles stephensi, Ethiopia, Malaria, Horn of Africa

\section{Background}

Malaria remains a leading global health concern with over 250 million cases reported yearly [1]. In Ethiopia, even though there has been steady progress in the reduction of malaria [2], 1.5 million cases were reported in 2018 [1]. Developing effective malaria control strategies in Ethiopia require knowledge of local mosquito vector species [3]. One threat to continued progress against malaria is the expansion of vectors into new areas. The South Asian vector Anopheles stephensi was recently discovered in Ethiopia and is raising concerns about the impact on malaria transmission in the country and the

\footnotetext{
*Correspondence: Meshesha_Managido@pmivectorlink.com

${ }^{1}$ Abt Associates, PMI VectorLink Ethiopia Project, Addis Ababa, Ethiopia

Full list of author information is available at the end of the article
}

rest of the Horn of Africa. Anopheles stephensi is a major malaria vector in South Asia and the Middle East, including the Arabian Peninsula [4], and is known to transmit both the major malaria parasite species Plasmodium falciparum and P. vivax $[5,6]$. The first report of An. stephensi in the Horn of Africa was from Djibouti in 2013 [7] and was recently confirmed to be persisting in the country [8]. Anopheles stephensi was detected in Ethiopia for the first time in 2016 in Kebridehar (Somali Region) but it remains unclear how broadly distributed the species is in the rest of the country [9].

Understanding the distribution of An. stephensi in Ethiopia is critical to evaluating the threat it poses to malaria control in Ethiopia and the rest of the Horn of Africa [9]. It is important during initial surveillance of a potential new vector to evaluate the accuracy of species 
identifications. Genetic analysis can be a useful complement to morphological identification to achieve optimal accuracy in species identification [10], particularly when

Table 1 Collection site altitude and geographical coordinates

\begin{tabular}{llll}
\hline Site & Region & Altitude (masl) & Coordinates \\
\hline Jigjiga & Somali & 1657 & $9^{\circ} 3^{\prime} 51^{\prime \prime} \mathrm{N}, 42^{\circ} 7^{\prime} 93^{\prime \prime} \mathrm{E}$ \\
Erer Gota & Somali & 1090 & $9^{\circ} 5^{\prime} 56^{\prime \prime} \mathrm{N}, 41^{\circ} 3^{\prime} 84^{\prime \prime} \mathrm{E}$ \\
Kebridehar & Somali & 532 & $6^{\circ} 7^{\prime} 38^{\prime \prime} \mathrm{N}, 44^{\circ} 2^{\prime} 77^{\prime \prime} \mathrm{E}$ \\
Godey & Somali & 294 & $5^{\circ} 9^{\prime} 49^{\prime \prime} \mathrm{N}, 43^{\circ} 5^{\prime} 53^{\prime \prime} \mathrm{E}$ \\
Degehabur & Somali & 1065 & $8^{\circ} 2^{\prime} 23^{\prime \prime} \mathrm{N}, 43^{\circ} 5^{\prime} 58^{\prime \prime} \mathrm{E}$ \\
Semera & Afar & 431 & $11^{\circ} 7^{\prime} 94^{\prime \prime} \mathrm{N}, 41^{\circ} 0^{\prime} 08^{\prime \prime} \mathrm{E}$ \\
Gewane & Afar & 617 & $10^{\circ} 1^{\prime} 66^{\prime \prime} \mathrm{N}, 40^{\circ} 6^{\prime} 46^{\prime \prime} \mathrm{E}$ \\
Awash Sebat Kilo & Afar & 916 & $89^{\prime} 89^{\prime \prime} \mathrm{N}, 40^{\circ} 1^{\prime} 64^{\prime \prime} \mathrm{E}$ \\
Dire Dawa & Dire Dawa & 1178 & $9^{\circ} 5^{\prime} 96^{\prime \prime} \mathrm{N}, 41^{\circ} 8^{\prime} 54^{\prime \prime} \mathrm{E}$ \\
Bati & Amhara & 2055 & $11^{\circ} 1^{\prime} 92^{\prime \prime} \mathrm{N}, 40^{\circ} 0^{\prime} 17^{\prime \prime} \mathrm{E}$
\end{tabular}

Abbreviation: masl, meters above sea level identifying a recently detected species. The objective of the study was to investigate the geographic distribution of An. stephensi in north eastern and eastern urban localities in Ethiopia using morphological and molecular identification of wild-caught Anopheles.

\section{Methods}

\section{Survey sites}

Anopheles stephensi surveys were conducted from August to November 2018 in ten selected urban sites situated in a climatic zone of either tropical, hot semi-arid or desert with an elevation range of 294 to 2055 meters above sea level. The localities included five in Somali region, three in Afar, one in Amhara region, and Dire Dawa city (Table 1, Fig. 1).

The areas have mean annual temperatures of about $20{ }^{\circ} \mathrm{C}$ to $30{ }^{\circ} \mathrm{C}$ and a mean annual rainfall from 200 to $900 \mathrm{~mm}$. There is a smaller rainy season between March and May, followed by a longer period between July and October [11].

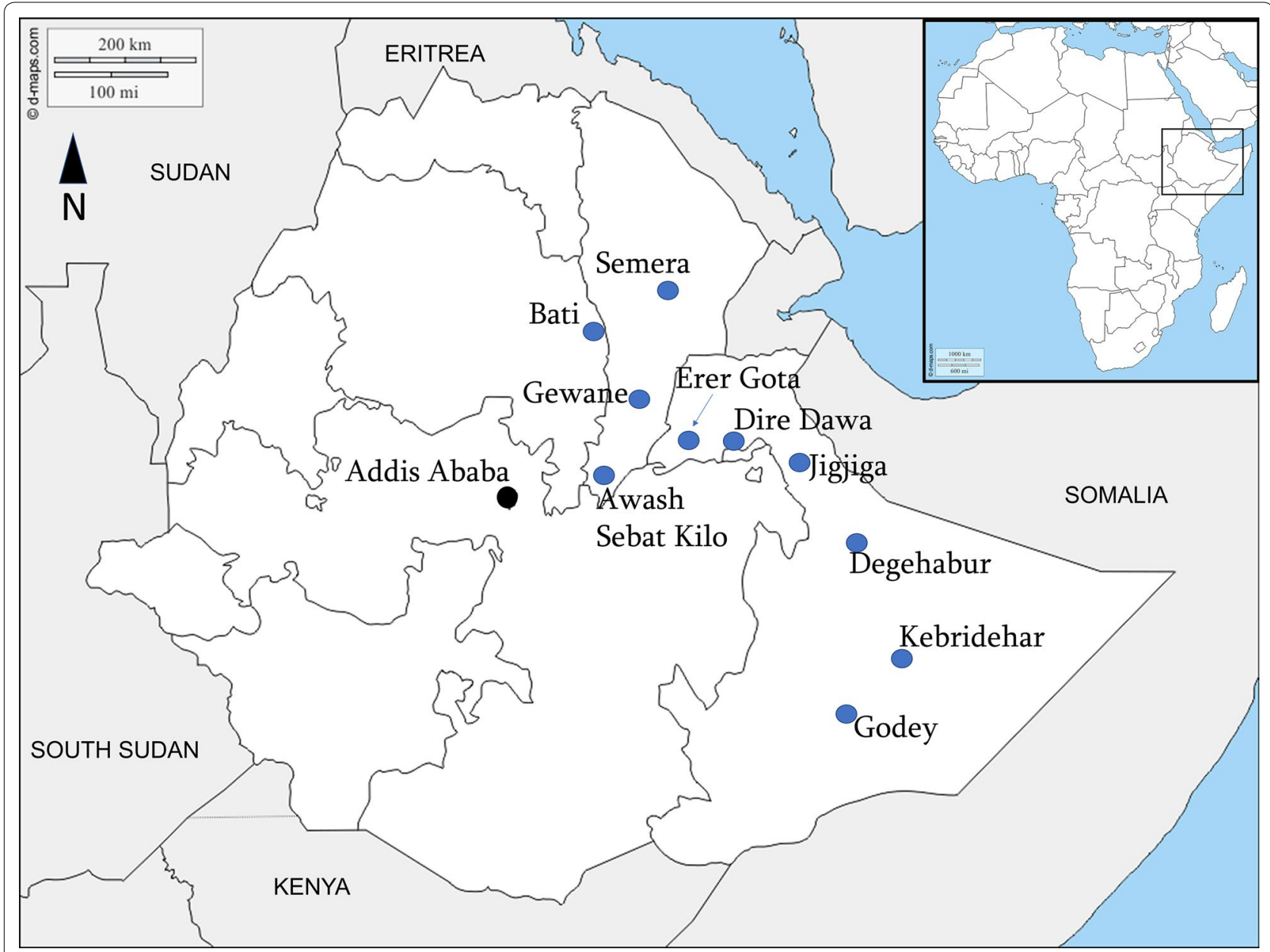

Fig. 1 Map of study sites in Ethiopia 


\section{Sampling of An. stephensi}

For the purpose of identification of An. stephensi and consequently to determine its presence in the study sites, free flying adult sampling was done together with raising of adults from larval and pupal collections.

\section{Adult sampling}

The entomological methods to sample adult mosquitoes were pyrethrum spray sheet collections (PSC) and Centers for Disease Control (CDC) light traps. In each site, PSC was conducted in 30 houses and CDC light traps were set in 20 houses for one night per house. Prior to PSC operation, consent was obtained from heads of households and PSC activities were conducted from 6:30 h to $8: 00 \mathrm{~h}$ mostly in sleeping rooms of big houses and additionally in living rooms of small houses. Houses for PSC were prepared following the WHO standard guidelines. All food and drink was removed, children and small animals made to stay outdoors, and non-removable items together with the floor were covered with pieces of white cloth sheet. All eaves, openings in windows and other mosquito exit points were blocked with pieces of cloth, as much as possible. Two operators, one inside and the other outside of the room/house were assigned to spray an aerosol insecticide (Baygon, SC Johnson \& Son Inc, Racine, Wisconsin, USA). Wearing a protective nose mask, the operator who was outside the house sprayed the aerosol walking around the house to drive in escapee mosquitoes while the other operator sprayed the insecticide in the whole room by moving from left to right of the door. Then the room/house was closed for ten minutes and knocked down mosquitoes were collected from the ground cloth using forceps and placed in Petri dishes.

CDC light trap collections were made from 18:00 $\mathrm{h}$ to 6:00 h. A CDC light trap was hung $1.5 \mathrm{~m}$ above the floor and close to a sleeping location where the occupants are protected with LLINs. Trapped mosquitoes were transferred from collection bag to cages. Those alive were killed with chloroform.

PSC and CDC light trap sampled mosquitoes were sorted into culicines and Anopheles. The revised morphological key of Gillies \& Coetzee [12] together with a key prepared by Maureen Coetzee (unpublished) were used to discriminate An. stephensi from other Anopheles spp. and An. arabiensis in particular. The morphological features used were: speckling on palps, base and apex of palp four with white scales; absence of upper proepisternal setae on the thorax and $3^{\text {rd }}$ main dark area (preapical dark spot) of wing vein R1 with no pale interruption; and having two pale spots on the second main dark area of the costa. Mosquitoes identified as An. stephensi were stored individually in Eppendorf tubes with silica gel to ensure the mosquitoes were kept dry for subsequent molecular analysis.

\section{Larval and pupal sampling}

Larvae and pupae of Anopheles were dipped from likely larval breeding habitats including man-made water containers, freshwater pools, stream margins, discarded tires, plastic containers and irrigation ditches. Water storage for household use and construction is common in the sites. These include metal and plastic tanks, cisterns, barrels and plastic sheets hung from polls. In the Somali region, water is stored in a container locally called "Birka" and it is constructed from cement and stone.

Larvae were reared in field insectaries using water taken from breeding habitats, feeding them with baking yeast and exposing them to sunlight during the day. Pupae were transferred into adult emergence cages and adults were identified to species using the keys described above; the specimens of An. stephensi were preserved as described above.

\section{Molecular identification of species}

To confirm morphological species identifications, a subset of the An. stephensi specimens were molecularly characterized. Additional An. gambiae (s.l.) specimens were also analyzed as controls for comparison. Species identification was completed using two approaches: (i) PCR endpoint assay utilizing the internal transcribed spacer 2 (ITS2) locus; and (ii) sequencing portions of ITS2 and cytochrome $c$ oxidase subunit 1 (cox1). ITS2 endpoint assay was performed as previously described [13] using the primers $5.8 \mathrm{SB}\left(5^{\prime}\right.$-ATG CTT AAA TTT AGG GGG TAG TC-3') and 28SC (5'-GTC TCG CGA CTG CAA CTG-3') and the following modifications: final reagent concentrations and components were $0.5 \mu \mathrm{M}$ for each primer; $1 \times$ Promega GoTAQ HotStart master mix (Promega, Madison, Wisconsin, USA); and water for a total reaction volume of $25 \mu \mathrm{l}$. The temperature protocol was performed as follows: $95{ }^{\circ} \mathrm{C}$ for $1 \mathrm{~min}, 30$ cycles of $96{ }^{\circ} \mathrm{C}$ for $30 \mathrm{~s}, 48{ }^{\circ} \mathrm{C}$ for $30 \mathrm{~s}, 72{ }^{\circ} \mathrm{C}$ for $1 \mathrm{~min}$, and a final extension step at $72{ }^{\circ} \mathrm{C}$ for $10 \mathrm{~min}$. Anopheles stephensi specimens were identified by visualization of 522-bp band with gel electrophoresis; non-An. stephensi specimens do not amplify and no band is present. Portions of the ITS2 and $\operatorname{cox} 1$ loci were also amplified for sequencing using previously detailed methods [14]. PCR products were sequenced using Sanger technology with ABI BigDyeTM terminator v3.1 chemistry (Thermofisher Scientific, Santa Clara, CA, USA) according to the manufacturer's recommendations and run on a 3130 Genetic Analyzer (Thermo Fisher Scientific). Sequences were cleaned and analyzed using CodonCode (CodonCode Corporation, Centerville, MA, USA). ITS2 and cox 1 sequences from 
Anopheles specimens were submitted as queries to the National Center for Biotechnology Information's (NCBI) Basic Local Alignment Search Tool (BLAST) [15] against the nucleotide collection in NCBI's GenBank under default parameters (max high-scoring segment pairs (HSP) 250, expect threshold 10, word size 28, optimized for highly similar hits, not specific to any organism). The Anopheles subject sequences from NCBI that formed HSP with the queries were used as the basis of species identification. The percent of species correctly identified using morphology was calculated using the molecular data for comparison.

\section{Plasmodium detection}

Wild-caught adult $A n$. stephensi were screened for $P$. falciparum and P. vivax DNA as an indication of Plasmodium infection. PCR amplification targeted the SSU RNA gene with species-specific primers using a previously published approach [16]. Presence of a band was a positive indication of Plasmodium DNA in the sample. Plasmodium falciparum and P. vivax SSU RNA positive controls were included for each reaction set (Microbiologics, St. Cloud, MN, USA).

\section{Results}

A total of 82 adult An. stephensi from 300 PSCs and 200 CDC light traps were collected in 7 of the 10 sites. The sites with no adult collections were Jigjiga, Awash Sebat Kilo and Bati (Table 1). Of the 82 adults $81.7 \%(n=67)$ were from PSC and the remaining $18.3 \%(n=15)$ were from CDC light traps. The majority of An. stephensi sampled using PSC were from Semera and Erer and that of CDC were from Degehabur. Larval and pupal collections yielded 2149 adult An. stephensi from all the sites confirming the presence of immature stages (Table 2).

Larval breeding habitats included concrete water cisterns, discarded tires, water tanks, steel drums, plastic sheet water storage at construction sites, discarded buckets, abandoned car wash and discarded vehicle part (Fig. 2). The potential breeding containers in each of the sites are presented in Table 3. However, since this was a onetime cross-sectional survey, the likelihood of missing other breeding sites is inevitable. Moreover, the containers found without larvae at the time of the survey might be positive another time because of the seasonality of the population of An. stephensi. Larvae and pupae of An. stephensi and Aedes aegypti were visually detected but not recorded. PCR endpoint assay was performed, and successful PCR products were obtained for 130 out of 133 Anopheles specimens. With the PCR endpoint assay, 119 specimens were identified as $A n$. stephensi and 11 specimens were identified as non- $A n$. stephensi. Sequencing of portions of the ITS 2 and cox 1 loci was also completed
Table 2 Number of adult An. stephensi collections from PSC, CDC traps, and larval and pupal collections

\begin{tabular}{|c|c|c|c|c|c|}
\hline \multirow[t]{3}{*}{ Region } & \multirow[t]{3}{*}{ Site } & \multicolumn{4}{|c|}{ Method of collections and An. stephensi } \\
\hline & & PSC & CDC & $\begin{array}{l}\text { Adults raised } \\
\text { from larvae and } \\
\text { pupae }\end{array}$ & Total \\
\hline & & $n(\%)$ & $n(\%)$ & $n(\%)$ & \\
\hline \multirow[t]{5}{*}{ Somali } & Jigjiga & $0(0)$ & $0(0)$ & $18(100)$ & 18 \\
\hline & Erer Gota & $14(9.8)$ & $0(0)$ & $129(90.2)$ & 143 \\
\hline & Kebridehar & $3(0.4)$ & $0(0)$ & $700(99.6)$ & 703 \\
\hline & Godey & $2(0.6)$ & $0(0)$ & $340(99.4)$ & 342 \\
\hline & Degehabur & $1(0.6)$ & $13(7.2)$ & $166(92.2)$ & 180 \\
\hline \multirow[t]{3}{*}{ Afar } & Semera & $38(12.7)$ & $1(0.3)$ & $260(87.0)$ & 299 \\
\hline & Gewane & $6(5.5)$ & $1(0.9)$ & $102(93.6)$ & 109 \\
\hline & $\begin{array}{l}\text { Awash Sebat } \\
\text { Kilo }\end{array}$ & $0(0)$ & $0(0)$ & $26(100)$ & 26 \\
\hline Dire Dawa & Dire Dawa & $3(1.1)$ & $0(0)$ & 277 (98.9) & 280 \\
\hline Amhara & Bati & $0(0)$ & $0(0)$ & 135 (100) & 135 \\
\hline Total & & 67 & 15 & 2149 & 2231 \\
\hline
\end{tabular}

Abbreviation: $\mathrm{n}$, number collected

and successful sequencing was completed for 118 Anopheles specimens. BLAST analysis of Anopheles sequences confirmed the positive detection of $A n$. stephensi at all ten sites. Sequence-based species identification was mostly consistent with ITS2 endpoint assay results, except for a single specimen that was morphologically identified as An. gambiae (s.l.), identified as non-An. stephensi with the endpoint assay, but sequencing detected An. stephensi. BLAST analysis of ITS2 sequences further identified all the non- $A n$. stephensi specimens as $A n$. arabiensis.

We compared the morphological identification to the ITS2 PCR endpoint assay results. Of the 130 Anopheles, 124 were classified as An. stephensi and six as An. gambiae (s.l.) based on morphology. Five of the 124 (4.0\%) morphologically identified An. stephensi were not confirmed to be An. stephensi with the PCR endpointassay. All morphologically identified An. gambiae (s.l.) (sequence-confirmed An. arabiensis) that were successfully amplified were also identified as non-An. stephensi with the ITS2 PCR endpoint assay.

All 82 wild-caught adult $A n$. stephensi were screened for P. falciparum or P. vivax infection. No Plasmodium DNA was detected in any of the specimens.

\section{Discussion}

This survey confirms that An. stephensi is distributed broadly in eastern Ethiopia. These data, taken with previous reports of An. stephensi in Kebridehar in 2016 [9], confirm that $A n$. stephensi is established in this region. 

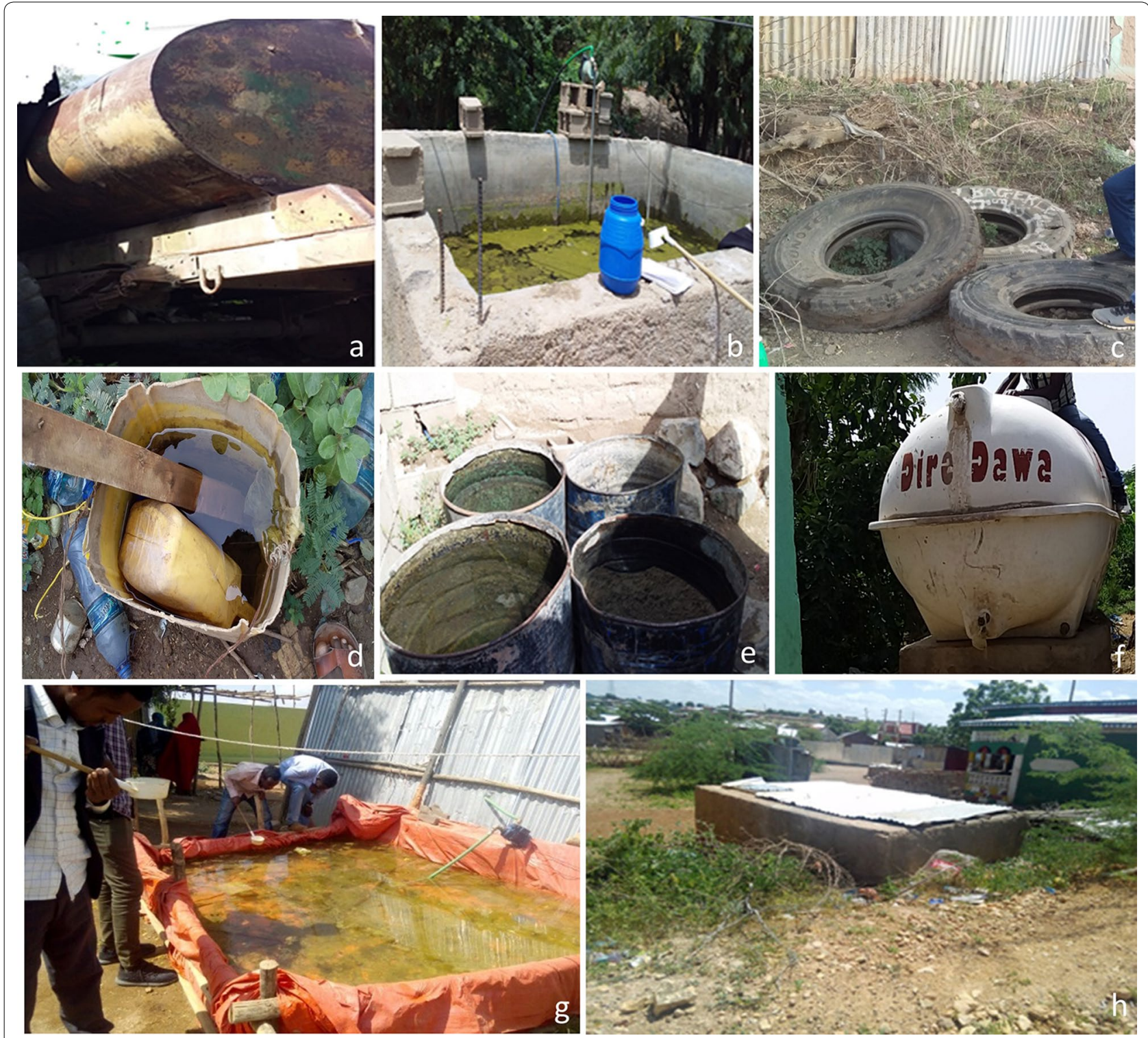

Fig. 2 Examples of breeding sites where An. stephensi larvae and pupae were found: a water tank trucks; $\mathbf{b}$ construction water storage reservoirs; $\mathbf{c}$ discarded tires; $\mathbf{d}$ buckets; $\mathbf{e}$ steel drums; $\mathbf{f}$ water tanks; $\mathbf{g}$ temporary water storage reservoirs; $\boldsymbol{h}$ birkas

To our knowledge, this is the first evidence for the presence of adult An. stephensi in multiple regions in Ethiopia, where it might transmit malaria. The widespread presence of An. stephensi in Ethiopia along with Djibouti suggests that neighboring countries, such as Sudan, South Sudan, Eritrea, Somalia and Kenya, should also enhance surveillance. Given that An. stephensi in Djibouti was found to carry both $P$. falciparum and $P$. vivax $[7,8]$, there is potential for these parasites to be observed in Ethiopia; thus, malaria control strategies should now consider the potential for the established An. stephensi to transmit malaria.
The presence of An. stephensi was confirmed using both morphological and molecular methods. While morphology was mostly consistent with molecular approach (119/124 correctly identified An. stephensi), there were a few instances of incorrect identification based on morphology highlighting the risk for the misidentification of specimens. As more vector surveillance programmes in Africa incorporate An. stephensi into their morphological keys, molecular data can be helpful with the evaluation of the successful training in An. stephensi morphological identification. This is particularly important at this beginning phase of An. stephensi surveillance as field 


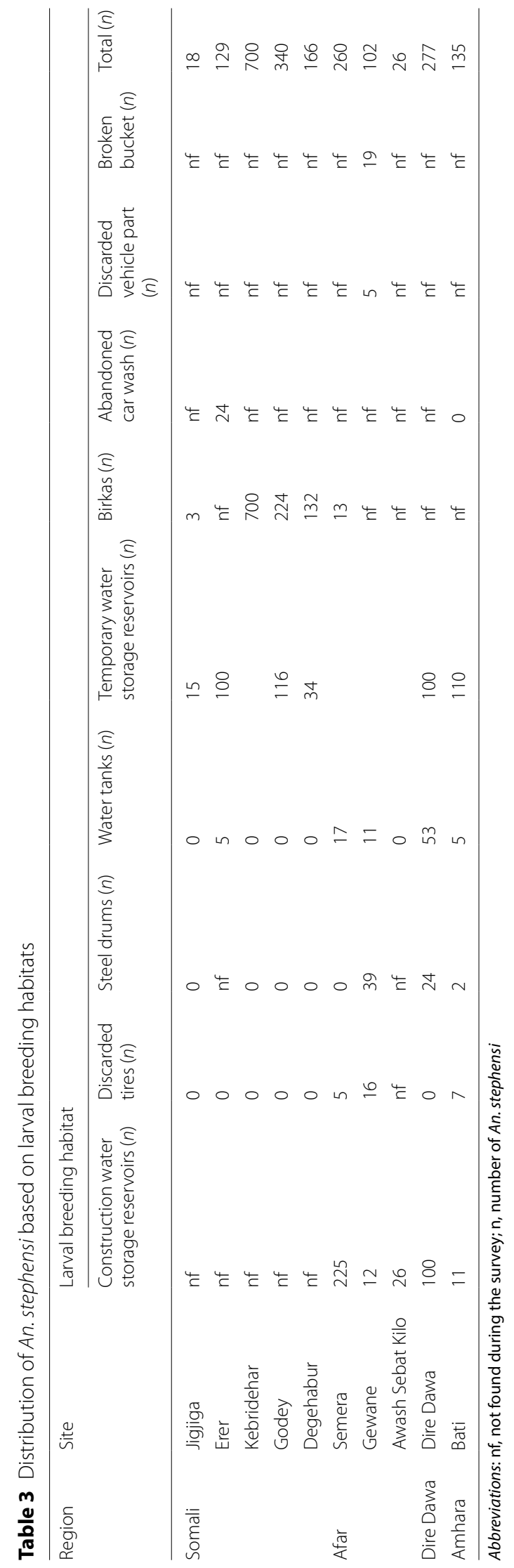


technicians will be adapting to detecting An. stephensi. We incorporated two molecular approaches, one of which, the end-point assay using primers designed by Djadid et al. [13] is more feasible in resource-limited settings. We found that this assay was mostly consistent with the sequence data and has to potential be integrated into current PCR based assays that focus on the detection of members of the An. gambiae complex, the most common malaria vectors in Africa.

While we have confirmed the broad distribution of An. stephensi in eastern Ethiopia, the distribution in the western part of the country is yet to be determined. West Ethiopia has had more consistent surveillance of malaria vectors than the eastern, due to the burden of the disease there; however, previously used trapping methods may limit the ability to detect An. stephensi. The current trapping techniques that rely heavily on $\mathrm{CDC}$ light traps may limit the ability to detect An. stephensi, given the low number of An. stephensi caught with CDC light traps at most sites in this study. Additional studies on the breeding, feeding, and resting behavior of An. stephensi can provide crucial information that can be applied to enhance future surveillance efforts in west and eastern Ethiopia.

Several additional areas of query need to be pursued further to better inform vector control efforts. No study has been published to confirm that the Ethiopian $A n$. stephensi can or does transmit Plasmodium. Both field confirmation of infected An. stephensi and laboratory infections are helpful approaches evaluating this information. In the present study, the 82 wild-caught $A n$. stephensi were screened for both $P$. falciparum and $P$. vivax using PCR, and Plasmodium was not detected. This is not unexpected as the regions included in this study report low malaria transmission, so a much larger sample size would be required to detect Plasmodium infection in An. stephensi. Future surveillance will continue to screen for Plasmodium using both a PCR-based and circumsporozoite protein enzyme-linked immunosorbent assay (ELISA).

The surveillance of An. stephensi to this point has been conducted in short time spans, with limited ability to assess changes in An. stephensi population size over time. We will be repeating collections in multiple sites in eastern Ethiopia to provide crucial information about how the population is changing year to year. This information will be particularly important as new vector control interventions are rolled out to evaluate their effectiveness. Insecticide resistance has been reported in the dominant malaria vector An. arabiensis in Ethiopia [17], but insecticide resistance status in $A n$. stephensi is unknown. Investigations into insecticide resistance, the molecular mechanisms behind resistance, and potential genetic markers that can be used for surveillance are ongoing.

One question that remains unanswered relates to the origin of An. stephensi in Ethiopia. Previous phylogeographic analysis revealed the closest $\operatorname{cox} 1$ sequence similarity of the Ethiopia An. stephensi found in Kebridehar to a specimen from Pakistan [9]. Phylogeographic analysis including sequencing from recent global $A n$. stephensi collections using multiple loci or whole genome sequences can help identify the exact origin of the $A n$. stephensi in the Horn of Africa and how it has spread throughout the region. This information will support efforts to prevent further introduction and spread of An. stephensi.

An ancillary observation during the surveillance was the detection of larvae of the dengue vector Ae. aegypti together with An. stephensi larvae, suggesting that these two vectors share larval habitats. Dengue is a growing public health concern in Ethiopia, particularly in eastern Ethiopia, where major outbreaks were reported in 2013 [18, 19] and 2015 [20]. With the finding of Ae. aegypti larvae with An. stephensi, we can consider integrated vector control to target both An. stephensi and Ae. aegypti. This would be a cost-effective approach to reducing both malaria and dengue virus transmission. Future surveillance in eastern Ethiopia will work towards determining relative abundance of Ae. aegypti larvae at An. stephensi breeding sites.

\section{Conclusions}

We confirmed that An. stephensi is widely distributed and established in eastern Ethiopia. Studies are ongoing to evaluate the distribution in the rest of the country and the potential risk for An. stephensi to change the malaria transmission landscape in the country and the rest of the African continent. Cross country cooperation and collaborations are needed to effectively address this potential global health concern.

\section{Abbreviations}

BLAST: Basic Local Alignment Search Tool; CDC: Centers for Disease Control and Prevention; cox1: cytochrome $c$ oxidase subunit 1; DNA: deoxyribonucleic acid; ELISA: enzyme-linked immunosorbent assay; HSP: high-scoring segment pairs; ITS2: internal transcribed spacer 2; NCBI: National Center for Biotechnology Information; PCR: polymerase chain reaction; PMI: US President's Malaria Initiative; PSC: pyrethrum spray catch; RNA: ribonucleic acid; SSU: small subunit; USAID: United States Agency of International Development; WHO: World Health Organization.

\section{Acknowledgements}

Cecilia Flately (Technical Programme Manager, Abt Associates, PMI VectorLink Project, Rockville, MD, USA) and Getinet Awoke (Environmental Compliance Officer, Abt Associates, PMI VectorLink Project, Addis Ababa, Ethiopia) are thanked for their assistance to the project. 


\section{Authors' contributions}

MB, PM, DD, SC, MM, KG, SI and TC developed the conception and design of the study. MB, GY, DG and SY collected the field data and identified mosquito samples. KL, DJ, SHC, JS and TC conducted and interpreted the laboratory analysis. MB and TC analyzed the data. MB, SI and TC contributed to the writing of the paper. All authors read and approved the final manuscript.

\section{Funding}

The US President's Malaria Initiative provided funding to PMI VectorLink (Abt Associates) for the training, collection, and identification of mosquitoes. SI was funded by PMI. TC was funded by Baylor University.

\section{Availability of data and materials}

Data supporting the conclusions of this article are included within the article. All data generated or analysed during this study are available from the corresponding author upon request.

\section{Ethics approval and consent to participate}

Wild mosquitoes used for this study were collected from dwellings and animal houses, following homeowners'verbal consent. The study protocol was reviewed by the Centers for Disease Control and Prevention, USA, and deemed to be non-human research (2017-227).

\section{Consent for publication}

Not applicable.

\section{Competing interests}

The authors declare that they have no competing interests.

\section{Author details}

${ }^{1}$ Abt Associates, PMI VectorLink Ethiopia Project, Addis Ababa, Ethiopia. ${ }^{2}$ Abt Associates, PMI VectorLink Project, Rockville, MD, USA. ${ }^{3}$ Dire Dawa University, Dire Dawa, Ethiopia. ${ }^{4}$ Jigjiga University, Jigjiga, Ethiopia. ${ }^{5}$ US President's Malaria Initiative (PMI), Addis Ababa, Ethiopia. ${ }^{6}$ United States Agency for International Development (USAID), Addis Ababa, Ethiopia. ${ }^{7}$ Division of Parasitic Diseases and Malaria, Center for Global Health, Centers for Disease Control and Prevention, Atlanta, GA, USA. ${ }^{8}$ Bureau for Global Health, Office of Infectious Disease, Malaria Division, USAID, Arlington, VA, USA. ${ }^{9}$ University of North Carolina at Charlotte, Charlotte, NC, USA. ${ }^{10}$ Baylor University, Waco, TX, USA.

Received: 24 October 2019 Accepted: 9 January 2020

Published online: 20 January 2020

\section{References}

1. WHO. World Malaria Report 2018. Geneva: World Health Organization; 2018. https://www.who.int/malaria/publications/world-malaria-repor t-2018/en. Accessed 19 Nov 2018.

2. Deribew A, Dejene T, Kebede B, Tessema GA, Melaku YA, Misganaw A, et al. Incidence, prevalence and mortality rates of malaria in Ethiopia from 1990 to 2015: analysis of the global burden of diseases 2015. Malar J. 2017;16:271.

3. Animut A, Lindtjørn B. Use of epidemiological and entomological tools in the control and elimination of malaria in Ethiopia. Malar J. 2018;17:26.

4. Sinka ME, Bangs MJ, Manguin S, Chareonviriyaphap T, Patil AP, Temperley $\mathrm{WH}$, et al. The dominant Anopheles vectors of human malaria in the AsiaPacific region: occurrence data, distribution maps and bionomic précis. Parasit Vectors. 2011:4:89.
5. Korgaonkar NS, Kumar A, Yadav RS, Kabadi D, Dash AP. Mosquito biting activity on humans and detection of Plasmodium falciparum infection in Anopheles stephensi in Goa, India. Indian J Med Res. 2012;135:120.

6. Thomas S, Ravishankaran S, Justin NJ, Asokan A, Mathai MT, Valecha N, et al. Resting and feeding preferences of Anopheles stephensi in an urban setting, perennial for malaria. Malar J. 2017;16:111.

7. Faulde MK, Rueda LM, Khaireh BA. First record of the Asian malaria vector Anopheles stephensi and its possible role in the resurgence of malaria in Djibouti, Horn of Africa. Acta Trop. 2014;139:39-43.

8. Seyfarth M, Khaireh BA, Abdi AA, Bouh SM, Faulde MK. Five years following first detection of Anopheles stephensi (Diptera: Culicidae) in Djibouti, Horn of Africa: populations established - malaria emerging. Parasitol Res. 2019;118:725-32.

9. Carter TE, Yared S, Gebresilassie A, Bonnell V, Damodaran L, Lopez K, et al. First detection of Anopheles stephensi Liston, 1901 (Diptera: Culicidae) in Ethiopia using molecular and morphological approaches. Acta Trop. 2018;188:180-6.

10. Lobo NF, St Laurent B, Sikaala CH, Hamainza B, Chanda J, Chinula D, et al. Unexpected diversity of Anopheles species in eastern Zambia: implications for evaluating vector behavior and interventions using molecular tools. Sci Rep. 2015;5:17952.

11. IRI/LDEO Climate Data Library. Palisades, NY: Lamont-Doherty Earth Observatory (LDEO) Climate Group, Columbia University. https://iridl Ideo.columbia.edu/maproom/Global/Climatologies/Select_a_Point.html. Accessed 1 Sep 2019.

12. Gillies MT, Coetzee M. A Supplement to the Anophelinae of Africa South of the Sahara (Afrotropical Region). Johannesburg: Publications of the South African Institute for Medical Research, Johannesburg; 1987.

13. Djadid ND, Gholizadeh S, Aghajari M, Zehi AH, Raeisi A, Zakeri S. Genetic analysis of rDNA-ITS2 and RAPD loci in field populations of the malaria vector, Anopheles stephensi (Diptera: Culicidae): implications for the control program in Iran. Acta Trop. 2006;97:65-74.

14. Carter TE, Yared S, Hansel S, Lopez K, Janies D. Sequence-based identification of Anopheles species in eastern Ethiopia. Malar J. 2019;18:135.

15. Altschul SF, Gish W, Miller W, Myers EW, Lipman DJ. Basic local alignment search tool. J Mol Biol. 1990;215:403-10.

16. Snounou G, Viriyakosol S, Jarra W, Thaithong S, Brown KN. Identification of the four human malaria parasite species in field samples by the polymerase chain reaction and detection of a high prevalence of mixed infections. Mol Biochem Parasitol. 1993;58:283-92.

17. Messenger LA, Shililu J, Irish SR, Anshebo GY, Tesfaye AG, Ye-Ebiyo Y, et al. Insecticide resistance in Anopheles arabiensis from Ethiopia (2012-2016): a nationwide study for insecticide resistance monitoring. Malar J. 2017; 16:469.

18. Woyessa AB, Mengesha M, Kassa W, Kifle E, Wondabeku M, Girmay A, Kebede A, Jima D. The first acute febrile illness investigation associated with dengue fever in Ethiopia, 2013: a descriptive analysis. Ethiop J Health Dev. 2014;28:155-61.

19. Ahmed YM, Salah AA. Epidemiology of dengue fever in Ethiopian Somali region: retrospective health facility based study. Cent Afr J Public Health. 2016;2:51-6.

20. Degife LH, Worku Y, Belay D, Bekele A, Hailemariam Z. Factors associated with dengue fever outbreak in Dire Dawa administration city, October 2015, Ethiopia-case control study. BMC Public Health. 2019;19:650.

\section{Publisher's Note}

Springer Nature remains neutral with regard to jurisdictional claims in published maps and institutional affiliations. 